\title{
LOCAL CONTROL OF $(4,5,7,8-10)$-FILTRATION SNAKE ROBOT VIA CGA
}

\author{
Jaroslav Hrdina, Aleš Návrat, Petr Vašík and Radomil Matoušek \\ Brno University of Technology \\ Faculty of Mechanical Engineering \\ Technická 2, Brno 61669 \\ Czech Republic \\ $\{$ hrdina, navrat.a, vasik, matousek\}@fme.vutbr.cz
}

\begin{abstract}
We describe the local control of a (6-8)-link snake like robot endowed with omnidirectional wheels on two links (head and tail). All calculations including the position, direct kinematics, differential kinematics and inverse kinematics are described in the terms of CGA only.
\end{abstract}

Keywords: Robotic snake, conformal geometric algebra, nonholonomic kinematics

\section{Introduction}

Let a non-holonomic system be described by the appropriate dynamic (Pfaff) system of ODEs, which defines the vectors of admissible motion directions (w.r.t. the controlling parameters). These vectors together with the Lie bracket operation generate a Lie algebra corresponding to the original system. Consequently, a nonholonomic system is said to be locally controllable if the linear span of its appropriate Lie algebra generators is of the same dimension as the configuration space (Rachevsky-Chow Theorem). Furthermore, while generating the Lie algebra, one obtains a natural filtration (w.r.t. the number of Lie bracket applications). The elements of the higher filtration parts correspond to the motions, which can not be realized locally but are necessary for local controllability. A general interpretation of such motions is crucial for local controllability description and realization. The main goal of the talk is to employ an advanced mathematical tool of the Conformal Geometric Algebra (CGA) in the snake robot modeling in order to achieve the optimal control of particular solutions.

\section{Conformal geometric algebra - CGA}

Let $\mathbb{R}^{4,1}$ denote a vector space $\mathbb{R}^{5}$ equipped with the scalar product of signature $(4,1)$, let $\mathcal{C} l(4,1)$ denote the corresponding Clifford algebra, i.e. a free, associative and distributive algebra as a span of the set $\left\{e_{1}, e_{2}, e_{3}, e_{+}, e_{-}\right\}$ such that the following identities are satisfied:

$$
\begin{gathered}
e_{1}^{2}=e_{2}^{2}=e_{3}^{2}=e_{+}^{2}=1, e_{-}^{2}=-1, \\
e_{i} e_{j}=-e_{j} e_{i}, i \neq j, i, j \in\{1,2,3,+,-\} .
\end{gathered}
$$

In this case, we get $2^{5}=32$-dimensional vector space. Let us note that the norm in $\mathbb{R}^{4,1}$ can be understood as a vector square $x^{2}=\|x\|^{2}$. Now, we define two additional products on $\mathbb{R}^{4,1}$ based on the geometric one for any $u, v \in \mathbb{R}^{4,1}$, dot product and wedge product, respectively:

$$
u \cdot v=\frac{1}{2}(u v+v u), \quad u \wedge v=\frac{1}{2}(u v-v u)
$$

and thus the formula for the geometric product can be derived as

$$
u v=u \cdot v+u \wedge v
$$

Generally, the wedge (outer) product of two basis blades $E_{i}$ and $E_{j}$, with $k=\operatorname{gr}\left(E_{i}\right), l=\operatorname{gr}\left(E_{j}\right)$ is defined as

$$
E_{i} \wedge E_{j}:=\left\langle E_{i} E_{j}\right\rangle_{k+l}
$$

and the dot (inner) product is defined as

$$
E_{i} \cdot E_{j}= \begin{cases}\left\langle E_{i} E_{j}\right\rangle_{|k-l|} & i, j,>0 \\ 0 & i=0 \text { or } j=0\end{cases}
$$


where $\operatorname{gr}(E)$ is a grade of the basis blade $E$ and \langle\rangle$_{k}$ is the grade projection into the blade of the grade $k$. To work with CGA effectively, we have to define a new basis of $\mathbb{R}^{4,1}$ as a set $\left\{e_{1}, e_{2}, e_{3}, e_{0}, e_{\infty}\right\}$ such that $e_{0}=\frac{1}{2}\left(e_{-}+e_{+}\right)$and $e_{\infty}=\left(e_{-}-e_{+}\right)$. Consequently, the following properties hold:

$$
\begin{aligned}
e_{0}^{2} & =0, \quad e_{\infty}^{2}=0, \quad e_{\infty} e_{0}=-1-e_{-} \wedge e_{+}, \quad e_{0} e_{\infty}=-1+e_{-} \wedge e_{+}, \\
e_{\infty} e_{0} & =-e_{0} e_{\infty}-2 .
\end{aligned}
$$

In CGA, we can represent the basis geometric elements by the following multivectors from $\mathcal{C l}(4,1)$ :

$$
\begin{aligned}
\text { point } x & \rightsquigarrow Q=x+\frac{1}{2} x^{2} e_{\infty}+e_{0} \\
\text { sphere of radius } & \rightsquigarrow S=C-\frac{1}{2} r^{2} e_{\infty} \\
r \text { and center } C & \\
\text { point pair } Q_{1}, Q_{2} & \rightsquigarrow P=Q_{1} \wedge Q_{2} .
\end{aligned}
$$

In CGA (in fact in GA generally), any transformation of the element $O$ is realized by conjugation

$$
O \mapsto M O \tilde{M}
$$

where $M$ is the appropriate multivector from $\mathcal{C l}(4,1)$. For instance, the translation in the direction $t=t_{1} e_{1}+$ $t_{2} e_{2}+t_{3} e_{3}$ is realized by the multivector

$$
M:=T=1-\frac{1}{2} t e_{\infty}
$$

and the rotation around the axis $L$ by angle $\phi$ is realized by the multivector

$$
M:=R=\cos \frac{\phi}{2}-L \sin \frac{\phi}{2}
$$

where $L=a_{1} e_{2} e_{3}+a_{2} e_{1} e_{3}+a_{3} e_{1} e_{2}$. For more information refer to books [2, 8] or papers [3, 4].

\section{The flag structure}

The snake robot described in this paper consists of 6-8 rigid links of constant length 2 interconnected by motorized joints. Except for the first and the last, to each line, in the center of mass, a pair of wheels is attached to provide an important snake-like property that the ground friction in the direction perpendicular to the link is considerably higher than the friction of a simple forward move. In particular, this prevents the slipping sideways. To determine the actual position of a snake robot we need the set of 8-10 generalized coordinates

$$
q=\left(x, y, \theta, \Phi_{i}, i \in\{1, \ldots, N\}\right) \in M,
$$

where $N \in\{5,6,7\}$ and $M=\mathbb{R}^{2} \times\left(\mathbb{S}^{1}\right)^{N+1}$ which describes the configuration of the snake robot.

The control theory generally leads to a nonholonomic system

$$
\dot{q}=\sum_{i=1}^{m} u_{i} X_{i}(q), \quad q \in M
$$

where $X_{i}$ are vector fields on $M$, where $m \in \mathbb{N}, m<\operatorname{dim} M$. In particular, the controllability of the nonholonomic system is fully characterized by the properties of the Lie algebra generated by $X_{1}, \ldots, X_{m}$. We define

$$
\Delta^{1}=\operatorname{span}\left\{X_{1}, \ldots, X_{m}\right\}
$$

and $\Delta^{s+1}=\Delta^{s}+\left[\Delta^{1}, \Delta^{s}\right]$, where $\left[\Delta^{1}, \Delta^{s}\right]=\operatorname{span}\left\{[X, Y]: X \in \Delta^{1}, Y \in \Delta^{s}\right\}$. The Lie algebra $\bar{\Delta}$ generated by $X_{1}, \ldots, X_{m}$ is defined as

$$
\bar{\Delta}=\bigcup_{s \geq 1} \Delta^{s} .
$$

Let us note that our system satisfies Chow's condition, i.e. $\bar{\Delta}(q)=T_{q} M, \forall q \in M$ and the vectors at $q \in M$ form a flag of subspaces of $T_{q} M$, that is

$$
\Delta^{1}(q) \subset \Delta^{2}(q) \subset \cdots \subset \Delta^{r-1}(q) \subset \Delta^{r}(q)=T_{q} M
$$


where $r=r(p)$ is so-called degree of nonholonomy at $p$. Set $n_{i}(q)=\operatorname{dim} \Delta^{i}(q)$. The $r$-tuple of integers $\left(n_{1}(q), \ldots, n_{r}(q)\right)$ is called the growth vector at $q$. In our cases the growth vectors are

$$
(4,5,7,8),(4,5,7,9),(4,5,7,10)
$$

and the degree of nonholonomy is 4 . The structure of the flag may also be described by another sequence of integers. We define the weights at $q, w_{i}=w_{i}(q), i=1, \ldots, m$, by setting $w_{j}=s$ if $n_{s-1}(q)<j \leq n_{s}(q)$, where $n_{0}=0$. In our cases the weights are

$$
(1,1,1,1,2,3,3,4),(1,1,1,1,2,3,3,4,4),(1,1,1,1,2,3,3,4,4,4) .
$$

As the first and the last link are endowed with the omnidirectional wheels and do not affect the control, the controlling Lie algebra is of a special form, particularly it contains a two-dimensional center $\mathcal{Z}$. The flag structures on factor space $\bar{\Delta} / \mathcal{Z}$ are characterized by the growth vectors

$$
(2,3,5,6),(2,3,5,7),(2,3,5,8),
$$

where the last example is of full dimension as the corresponding P. Hall basis contains exactly 8 elements. As a result, we discuss all possibilities with the degree of nonholonomy equal to 4 after the center factorisation.

\section{Kinematics}

Note that a fixed coordinate system $(x, y)$ is attached. The points $p_{i}:=\left(x_{i}, y_{i}\right)$, denote the centers of mass of each link. To describe the robotic snake we use as a central object the set of point pairs

$$
\left(P_{0}, P_{2}, P_{4}, \cdots, P_{N}\right)
$$

where $P_{0}=Q_{0} \wedge Q_{1}, P_{1}=Q_{1} \wedge Q_{2}$ and $P_{N}=Q_{N} \wedge Q_{N+1}$, where $Q_{i}$ are the joint points and head and tail points. Consequently, the kinematic equations can be assessed and if we consider the projections

$$
Q_{i}=-\frac{\sqrt{P_{i} \cdot P_{i}}+P_{i}}{e_{\infty} \cdot P_{i}}, \quad Q_{i+1}=\frac{\sqrt{P_{i} \cdot P_{i}}+P_{i}}{e_{\infty} \cdot P_{i}}
$$

we are able to express the coordinates of every point from any point pair. The coordinates of particular position of link centers are the following

$$
p_{i}=P_{i} e_{\infty} \tilde{P}_{i} \text {, s.t. } \quad P_{i}=R_{\Phi_{i}} \cdots R_{\Phi_{1}} R_{\theta} T_{x, y} P_{i, 0} \tilde{T}_{x, y} \tilde{R}_{\theta} \tilde{R}_{\Phi_{1}} \cdots \tilde{R}_{\Phi_{i}}
$$

and, for the robotic snake initial position $x=y=\theta=\Phi_{i}=0$, the appropriate point pairs are denoted by $P_{i, 0}$ and calculated directly, e.g.

$$
\begin{aligned}
& P_{1,0}=\left(e_{0}\right) \wedge\left(2 e_{1}+2 e_{\infty}+e_{0}\right)=2 e_{0} e_{1}-2 e_{+} e_{-} \\
& P_{2,0}=\left(2 e_{1}+2 e_{\infty}+e_{0}\right) \wedge\left(4 e_{1}+8 e_{\infty}+e_{0}\right)=2 e_{0} e_{1}+8 e_{1} e_{\infty}-6 e_{+} e_{-}, \\
& P_{3,0}=\left(4 e_{1}+8 e_{\infty}+e_{0}\right) \wedge\left(6 e_{1}+18 e_{\infty}+e_{0}\right)=2 e_{0} e_{1}+24 e_{1} e_{\infty}-10 e_{+} e_{-}
\end{aligned}
$$

Now, the transformations corresponding to the generalized coordinates can be written as

$$
\begin{aligned}
T_{x, y} & =1-\frac{1}{2}\left(x e_{1}+y e_{2}\right) e_{\infty}, \\
T_{Q_{i}} & =1-\frac{1}{2} Q_{i} e_{\infty}, \\
R_{0} & =\cos \frac{\theta}{2}-L_{0} \sin \frac{\theta}{2}, \text { where } L_{0}=T_{x, y} e_{1} e_{2} \tilde{T}_{x, y}, \\
R_{i} & =\cos \frac{\Phi_{1}}{2}-L_{i} \sin \frac{\Phi_{1}}{2}, \text { where } L_{i}=T_{Q_{i}} e_{1} e_{2} \tilde{T}_{Q_{i}} .
\end{aligned}
$$

The direct kinematics for the snake robot is obtained similarly as the kinematics for serial robot arms [10]. In general, it is given by a succession of generalised rotations $R_{i}$ and it is valid for all geometric objects, including point pairs. A point pair $P$ in a general position is computed from its initial position $P_{0}$ as follows

$$
P=\prod_{i=1}^{n} R_{i} P_{0} \prod_{i=1}^{n} \tilde{R}_{n-i+1} .
$$




\section{Differential kinematics}

Unlike the fixed serial robot arms, we allow $R_{i}$ to be also a translation. We view translations as degenerate rotations. Then the differential kinematics is expressed by means of the total differential as follows

$$
d P=\sum_{j=1}^{n} \partial_{q_{j}}\left(\prod_{i=1}^{n} R_{i} P_{0} \prod_{i=1}^{n} \tilde{R}_{n-i+1}\right) d q_{j}
$$

Theorem. Let $c$ be a centre of a sphere $S$ (including a point pair as a $0 D$-sphere) whose final position is given by the kinematic chain (2). Then the differential kinematics of $c$ is given by

$$
\dot{c}=\sum_{j=1}^{n}\left[c \cdot L_{j}^{\prime}\right] d q_{j}
$$

Proof. See [4].

Concretly, we obtain the system

$$
\begin{aligned}
\dot{p_{1}} & =\left[p_{1} \cdot e_{1} e_{\infty}\right] \dot{x}+\left[p_{1} \cdot e_{2} e_{\infty}\right] \dot{y}+\left[p_{1} \cdot L_{0}\right] \dot{\theta} \\
\dot{p_{i}} & =\left[p_{2} \cdot e_{1} e_{\infty}\right] \dot{x}+\left[p_{2} \cdot e_{2} e_{\infty}\right] \dot{y}+\left[p_{i} \cdot L_{0}\right] \dot{\theta}+\left[p_{2} \cdot L_{1}\right] \dot{\Phi}_{1}+\cdots+\left[p_{2} \cdot L_{1}\right] \dot{\Phi}_{i},
\end{aligned}
$$

which in the matrix notation is of the form

$$
\dot{p}=J \dot{q}
$$

where $q$ are our coordinates $(1)$ and $J=\left(j_{k l}\right)$ is a matrix with the elements defined by scalar products of points and axes

$$
\begin{aligned}
j_{i 1} & =\left[p_{i} \cdot e_{1} e_{\infty}\right], j_{i 2}=\left[p_{i} \cdot e_{2} e_{\infty}\right] \\
j_{i k} & =\left[p_{i} \cdot L_{k-3}\right] \text { for } 3 \leq k<3+i \\
j_{i k} & =0 \text { for } 3+i \leq k
\end{aligned}
$$

As the wheels do not slip to the side direction, the velocity constraint condition is satisfied for each link $i$ and in terms of CGA can be written as

$$
\dot{p}_{i} \wedge P_{i} \wedge e_{\infty}=0
$$

Thus if we substitute (3) in (4), we obtain a system of linear ODEs, which has a simple Pfaff matrix form

$$
A \dot{q}=0
$$

where $A=\left(a_{i j}\right)$ is a matrix with the elements defined by

$$
a_{i k}=j_{i k} \wedge P_{i} \wedge e_{\infty}
$$

Note that the enteries of $A$ are multiples of $e_{1} e_{2} e_{+} e_{-}$. Taking the conjugate and multiplying with $e_{3} A$ can be considered simply as a matrix over the field of functions. For example, the solution of this system with respect to $\dot{\theta}$ parameterized by $\dot{x}, \dot{y}$, (i.e. $\dot{x}=t_{1}$ and $\dot{y}=t_{2}$ ) is of the form

$$
\dot{\theta}=-\frac{\left[p_{1} \cdot e_{1} e_{\infty}\right] \wedge P_{1} \wedge e_{\infty}}{\left[p_{1} \cdot L_{0}\right] \wedge P_{1} \wedge e_{\infty}} t_{1}-\frac{\left[p_{1} \cdot e_{2} e_{\infty}\right] \wedge P_{1} \wedge e_{\infty}}{\left[p_{1} \cdot L_{0}\right] \wedge P_{1} \wedge e_{\infty}} t_{2}
$$

If we denote by $e^{*}$ the dual to $e$ in CGA which is realized by the multiplication of the inverse unit pseudoscalar, the straightforward computation leads to $\left[p_{1} \cdot L_{0}\right] \wedge P_{1} \wedge e_{\infty}=2 e_{3}^{*}$, i.e. the solution always exists, because $\left(\left[p_{1} \cdot L_{0}\right] \wedge P_{1} \wedge e_{\infty}\right)^{-1}=-\frac{1}{2} e_{3}^{*}$.

The singular posture of the system is in the case that the wheel axes, i.e. lines perpendicular to each link containing the link center point, intersect in precisely one point or are parallel, see Figure 2 . In our setting this is one condition only because in CGA the parallel lines intersect in exactly one point which is $e_{\infty}$. It is 
easy to see that this happens in such case that all joints lie on a single circle, i.e. in CGA they satisfy a simple condition

$$
Q_{0} \wedge Q_{1} \wedge Q_{2} \wedge Q_{i}=0, \quad \forall i \in\{3, \ldots, N+1\} .
$$

Finally, note that the non-singular solution forms a 2-dimensional distribution which can be parametrized e.g. as follows:

$$
\dot{q}=G\left(\begin{array}{l}
t_{1} \\
t_{2}
\end{array}\right)
$$

where $G=\left(g_{i j}\right)$ is a control matrix. Thus if we consider the snake robot configuration space with coordinates (1) as manifold $M$, the solution above forms a set of vector fields $\left\{g_{1}, g_{2}, g_{3}, g_{4}\right\}$, such that $g_{3}, g_{4} \in \mathcal{Z}$.

It is clear, that the space $\operatorname{span}\left\{g_{1}, g_{2}\right\}$ determines the set of accessible spatial velocity vectors and thus, taking into account the vector field flows $\exp \left(t g_{1}\right), \exp \left(t g_{2}\right)$, the possible trajectories of the snake robot. On the other hand, due to non-commutativity of $\exp \left(t g_{1}\right), \exp \left(t g_{2}\right)$, the robot can move even along the flow of the Lie bracket by means of the composition

$$
\exp \left(-t g_{2}\right) \circ \exp \left(-t g_{1}\right) \circ \exp \left(t g_{2}\right) \circ \exp \left(t g_{1}\right)
$$

Extending this idea, the space $D_{q}$ of all movement directions at the point $q$ is given by all possible Lie brackets of $g_{1}(q)$ and $g_{2}(q)$ and the resulting vector fields. From the geometric control theory point of view, it is quite necessary that the dimension of $D_{q}$ is equal to the dimension of the tangent space $T_{q} M, q \in M$, which in our case is 6,7 and 8 , respectively. Note that this is the condition on the model local controllability given by the Rashevsky-Chow Theorem.

\section{$6 \quad$ Notes on the inverse kinematics}

Note that the geometric meaning of the inner product of two conformal vectors $U$ and $V$ is the following.

Table 1: Geometric meaning of the inner product

\begin{tabular}{|l|l|l|l|}
\hline$U \cdot V$ & Plane & Sphere & Point \\
\hline Plane & Angle between planes & Euclidean distance from center & Euclidean distance \\
\hline Sphere & Euclidean distance from center & Distance measure & Distance measure \\
\hline point & Euclidean distance & Distance measure & Euclidean distance \\
\hline
\end{tabular}

In our case, we have the set of point pairs $\left\{P_{0}, \ldots, P_{N}\right\}$ which determines the mechanism configuration uniquely. Using the notation of Section 4 , the set of the admissible points $\left\{Q_{0}, \ldots, Q_{N+1}\right\}$ with respect the set of point pairs $\left\{P_{0}, \ldots, P_{N}\right\}$ has to satisfy the equations

$$
Q_{i} \cdot Q_{i+1}=2,
$$

where $i \in\{0, \ldots, N\}$. A point in the configuration space $M$ is determined by the following direct computation:

$$
\begin{aligned}
x & =Q_{0} \cdot e_{1}, \\
y & =Q_{0} \cdot e_{2}, \\
\cos \theta & =\left(P_{1} \wedge e_{\infty}\right) \cdot\left(e_{0} \wedge e_{1} \wedge e_{\infty}\right), \\
\cos \phi_{i} & =\left(P_{i} \wedge e_{\infty}\right) \cdot\left(P_{i+1} \wedge e_{\infty}\right) .
\end{aligned}
$$

Various inverse problems can be solved by these equitations. For instance assume the position of the first and the last link is fixed (as it does not affect the control process) and all possible resulting configurations are computed. We shall use the property of the scalar product of a point $Q$ and a sphere $S$ that the number $\sqrt{2|Q \cdot S|}$ determines the Euclidean distance of $Q$ and the point of tangency on $S$ appropriate to the tangent containing $Q$. To avoid the singular initial positions we suggest to employ the assumption for small $\epsilon \in \mathbb{R}$

$$
2-\epsilon \leq Q_{i} \cdot\left(Q_{i+2}-2 e_{\infty}\right) \leq 2+\epsilon,
$$

which reads that the angel $\phi_{i}$ is in the vicinity of $\frac{\pi}{2}$ or less restrictive assumption

$$
\operatorname{sgn}\left(\left(P_{i} \wedge e_{\infty}\right) \cdot\left(P_{i+1} \wedge e_{\infty}\right)\right)=-\operatorname{sgn}\left(\left(P_{i} \wedge e_{\infty}\right) \cdot\left(P_{i+1} \wedge e_{\infty}\right)\right)
$$

which reads that the angle signum is changing in each joint.

Acknowledgement: The authors were supported by a grant of the Czech Science Foundation (GAČR) no. 17-21360S. 


\section{References}

[1] L. Gonzalez-Jimenez, O. Carbajal-Espinosa, A. Loukianov, and E. Bayro-Corrochano, Robust Pose Control of Robot Manipulators Using Conformal Geometric Algebra, Advances in Applied Clifford Algebras 24 (2), 533-552 (2014).

[2] Hildenbrand, D.: Foundations of Geometric Algebra Computing, Springer, Geometry and Computing, Vol. $8,9(2013)$.

[3] Hrdina, J., Návrat, A., Vašík, P.: 3-link robotic snake control based on CGA, Advances in Applied Clifford Algebras, 26(3) , 1069-1080 (2016).

[4] Hrdina, J. and Vašík, P.: Notes on differential kinematics in conformal geometric algebra approach, Recent Advances in Soft Computing, Advances in Intelligent Systems and Computing, vol. 378, pp. 363-374, Springer (2015).

[5] Liljebäck, P., Pettersen, K.Y. , Stavdahl, Ø. and Gravdahl, J.T.: Snake Robots, Modelling, Mechatronics and Control, Advances in Industrial Control, Springer, (2013).

[6] Matousek, R. and Návrat, A.: Trident snake control based on CGA, Recent Advances in Soft Computing, Advances in Intelligent Systems and Computing, vol. 378, pp. 375-385, Springer (2015).

[7] Murray, R. M., Zexiang, L. and Sastry, S.S.: A Mathematical Introduction to Robotic Manipulation, CRC Press (1994).

[8] Perwass, Ch.: Geometric Algebra with Applications in Engineering, Geometry and Computing, Vol. 4, Springer (2009).

[9] Selig, J.M.: Geometric Fundamentals of Robotics, Monographs in Computer Science, Springer (2004).

[10] Zamora-Esquivel, J. and Bayro-Corrochano, E.: Kinematics and diferential kinematics of binocular robot heads, Proceedings 2006 IEEE International Conference on Robotics and Automation, 4130-4135 (2006).

[11] Zamora-Esquivel, J. and Bayro-Corrochano, E.: Parallel Forward Dynamics: a geometric approach, Proceedings 2010 IEEE/RSJ International Conference on Intelligent Robots and Systems, 2377-2382 (2010). 